\title{
Retrosigmoid Suprameatal Approach for Resection of Petrotentorial Cerebellopontine Angle Meningioma: Operative Video and Technical Nuances
}

\author{
James K. Liu ${ }^{1}$ Vincent N. Dodson ${ }^{1}$ \\ ${ }^{1}$ Department of Neurological Surgery, Center for Cerebrovascular \\ and Skull Base Surgery, Rutgers University, New Jersey Medical \\ School, Neurological Institute of New Jersey, RWJ Barnabas Health, \\ Livingston and Newark, New Jersey, United States
}

\begin{abstract}
Address for correspondence James K. Liu, MD, Department of Neurological Surgery, Rutgers University, New Jersey Medical School, 90 Bergen Street, Suite 8100, Newark, New Jersey 07103, United States (e-mail: james.liu.md@rutgers.edu).
\end{abstract}

J Neurol Surg B 2019;80(suppl S3):S290-S291.

\begin{abstract}
Meningiomas are the second most common tumor to arise in the cerebellopontine angle. In this operative video atlas manuscript, the authors demonstrate a step-by-step technique for microsurgical resection of a petrotentorial meningioma in the cerebellopontine angle via a retrosigmoid suprameatal approach. Drilling of the hyperostotic suprameatal tubercle was necessary to gain access to the dural origin and anterior

Keywords

- retrosigmoid

- suprameatal

- petrotentorial

- cerebellopontine angle

- meningioma

- microsurgery petrosal extent of the tumor. The nuances of microsurgical and skull base technique are illustrated including microsurgical dissection of the tumor away from the brainstem and neurovascular structures, facial nerve preservation, and fat graft-assisted Medpor Titan reconstruction to prevent cerebrospinal fluid leakage. A gross total resection was achieved, and the patient was neurologically intact. In summary, the retrosigmoid suprameatal approach is an important strategy in the armamentarium for surgical management of petrotentorial meningiomas in the cerebellopontine angle. The link to the video can be found at: https://youtu.be/kwQP6BSYK7U.
\end{abstract}

Conflict of Interest None declared.

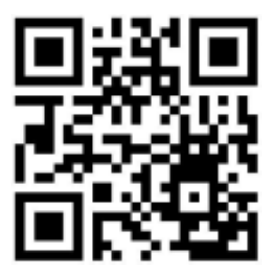

received

March 5, 2019

accepted

March 6, 2019

published online

April 22, 2019 www.thieme.com/skullbasevideos

www.thieme.com/jnlsbvideos
License terms

(c) $(1)$
DOI https://doi.org/

10.1055/s-0039-1685532. ISSN 2193-6331. (c) 2019 Georg Thieme Verlag KG Stuttgart · New York 


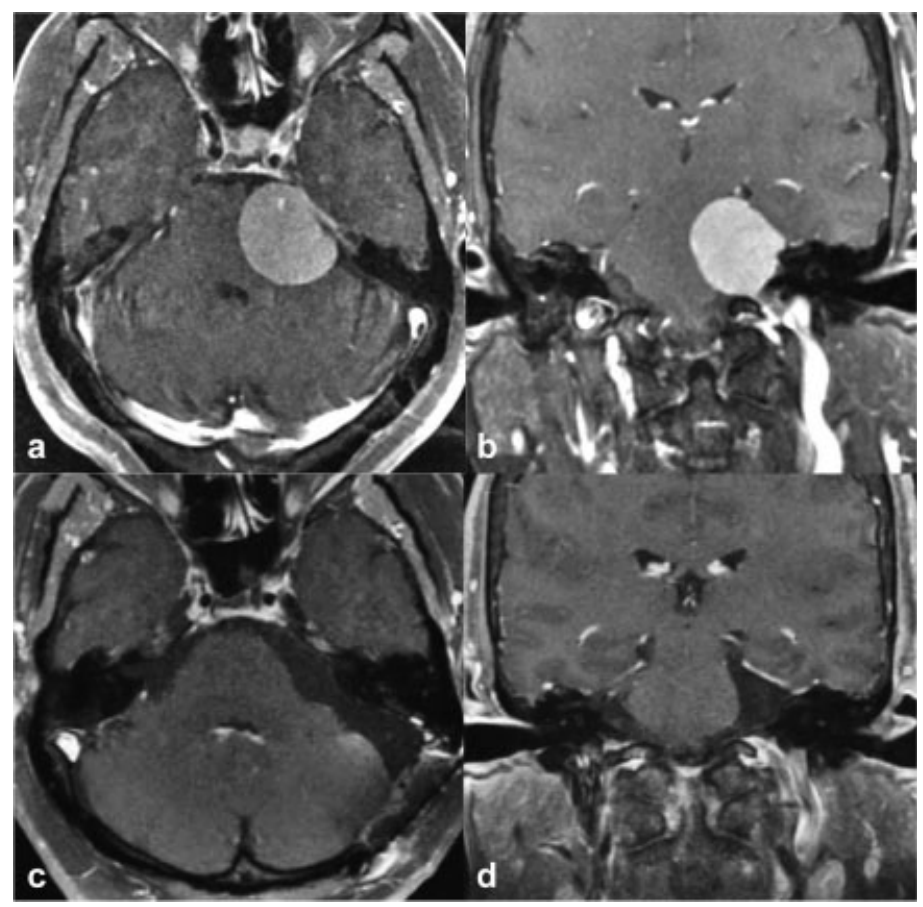

Fig. 1 Preoperative post-gadolinium T1-weighted MRI (a, axial view; b, coronal view) demonstrates left petrotentorial meningioma with brainstem compression. Postoperative MRI at 3 months (c, axial view; d, coronal view) shows no recurrence or residual tumor.

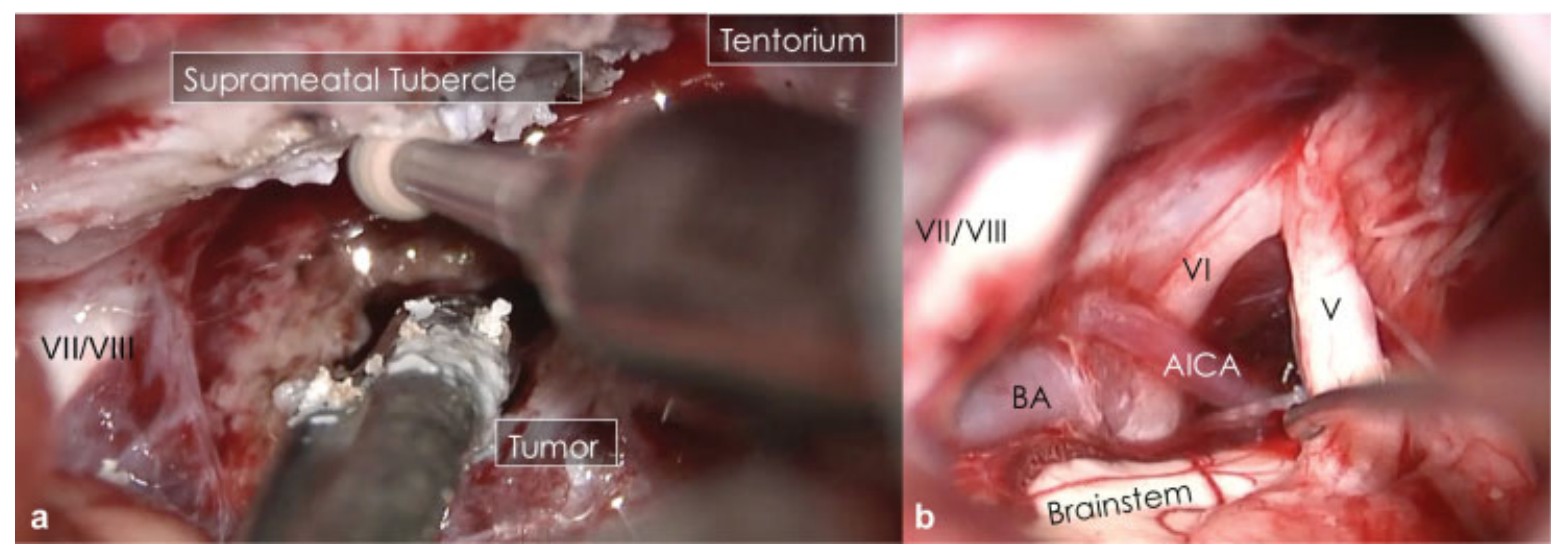

Fig. 2 (a) Intraoperative photograph showing drilling of the suprameatal tubercle to gain access to the anterior petrosal portion of the tumor. (b) View of resection bed exposing cranial nerves $V$ to VIII. 